\title{
Latest Higgs Physics Results from ATLAS
}

\author{
Jürgen Kroseberg ${ }^{\mathrm{a}}$, on behalf of the ATLAS Collaboration \\ ${ }^{a}$ Physikalisches Institut der Universität Bonn, Nussallee 12, D-53115 Bonn, Germany
}

\begin{abstract}
Selected recent results on Higgs boson physics obtained with the ATLAS experiment at the LHC are presented. These include preliminary evidence for the Higgs Yukawa interaction with fermions based on $\tau \tau$ final states, results from an updated combined analyses of Higgs boson couplings, new mass measurements, constraints on the total width, differential cross section measurements as well as the search for rare production and decay modes.
\end{abstract}

Keywords: Higgs, ATLAS, LHC

\section{Introduction}

The discovery of a previously unobserved particle with a mass of about $125 \mathrm{GeV}$ by the ATLAS [1] and CMS experiments at the LHC, which was announced in July 2012, provided an excellent candidate for the Higgs boson in the framework of the Standard Model of particle physics (SM). Evidence was seen in a combination of searches for three decay modes: $H \rightarrow \gamma \gamma$, $H \rightarrow Z Z^{*} \rightarrow 4 l$ and $H \rightarrow W W \rightarrow 2 \ell 2 v(l=e, \mu)$. The observed rates and event properties, based on data corresponding to an integrated luminosity of about $5 \mathrm{fb}^{-1}$ per experiment, were consistent with a single, narrow-width Higgs boson with production cross sections and branching fractions for the decays to bosons as predicted by the SM $[2,3]$.

Since then, further improved analyses have been performed on four to five times larger data sets in order to improve the sensitivity with respect to previously observed decay modes, to establish signals in other decay modes, in particular fermionic channels, and to extract information on other properties in addition to the mass of the new particle. In summer 2013, the ATLAS collaboration summarised their intermediate findings on the couplings [4] and evidence for the spin-0 nature [5] of the Higgs boson candidate, again focusing on the Higgs decay modes to boson pairs listed above.

In the following, a selection of more recent results is used to provide an overview of the current status of Higgs boson studies with the ATLAS experiment. The discussion focusses on the SM, although there is also a

Email address: kroseberg@uni-bonn.de (Jürgen Kroseberg) broad ATLAS Higgs physics program considering various SM extensions. All results use the complete ATLAS data recorded at a proton-proton centre-of-mass energy of $\sqrt{s}=8 \mathrm{TeV}$, which correspond to an integrated luminosity of $20.3 \mathrm{fb}^{-1}$; some of them include in addition 4.5-4.8 $\mathrm{fb}^{-1}$ of data obtained at $\sqrt{s}=7 \mathrm{TeV}$.

\section{Evidence for Decay into Fermions}

The initial observation of the Higgs boson candidate and the first published ATLAS results on its properties were based on decays into pairs of bosons. Since then, however, also direct evidence for Higgs Yukawa couplings to fermions has been found via a preliminary analyses

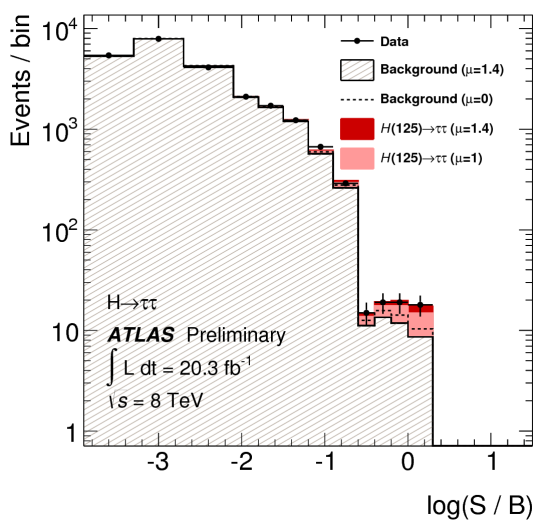

Figure 1: Preliminary evidence for the decay $H \rightarrow \tau \tau$ : event yields as a function of $\log (\mathrm{S} / \mathrm{B})$, where $\mathrm{S}$ (signal yield) and B (background yield) are taken from the corresponding bin of the distribution of the relevant BDT output discriminant [6]. 
of $\tau \tau$ final states [6]. All three combinations of leptonic and hadronic tau decays are considered and, for each of these final states, two categories are defined, the first targeting signal events produced via vector-boson fusion (VBF) by requiring at least two high- $p_{T}$ jets with a large separation in pseudorapidity and the other focusing on boosted Higgs boson topologies, which enriches mainly gluon-gluon fusion (ggF) Higgs boson production with additional jets and $\mathrm{VH}$ processes, where the Higgs boson is produced in association with a vector boson. Since the event characteristics and thus the composition of the relevant background contributions vary significantly between these eight categories, the data analysis is performed separately for each of them. The signal is extracted from the distribution of the output of boosted decision trees (BDTs) built from the invariant $\tau \tau$ mass and four to eight additional quantities. The resulting event yields in all BDT bins as a function of $\log (\mathrm{S} / \mathrm{B})$ are shown in Fig. 1. The statistical analysis yields a signal significance of $4.1 \sigma$ (with $3.2 \sigma$ expected), corresponding to a signal strength ${ }^{1}$ of $\mu=1.4_{-0.4}^{+0.5}$. The observed signal is compatible with a Higgs boson mass of $125 \mathrm{GeV}$, as demonstrated by Fig. 2 .

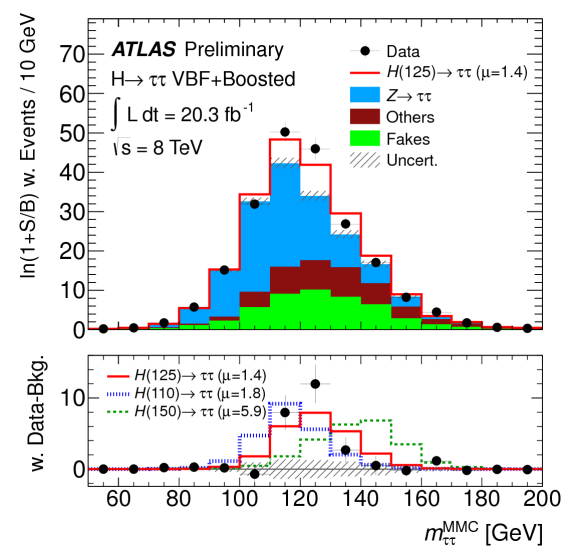

Figure 2: Distributions of the reconstructed invariant $\tau \tau$ mass, where events are weighted by $\ln (1+\mathrm{S} / \mathrm{B})$. Signal is shown stacked on background, with both predictions coming from the global fit yielding a signal strength of $\mu=1.4$. The bottom panel shows the difference between weighted data events and weighted background events (black points), compared to the weighted signal yields for different $m_{H}$ assumptions [6]

Searches for the decay $H \rightarrow b \bar{b}$ have not yet resulted in a significant evidence. A preliminary analysis [7], targeting Higgs production in association with vector bosons, yields observed (expected) 95\% C.L. limits of 1.4(1.3) times the SM prediction.

\footnotetext{
${ }^{1}$ For a given Higgs boson mass, the parameter $\mu$ is defined as the observed Higgs boson production strength normalised to the SM expectation.
}

\section{Updated Combined Analysis}

Fig. 3 summarises the measured signal strengths from analyses of different final states collected in [8].

The observed signal strength for a given channel is sensitive to the Higgs boson couplings to other particles through both the production and the decay of the Higgs boson. In order to test these couplings for the observed Higgs boson candidate, the data are re-analysed allowing for modifications in the coupling strengths. These modifications are implemented for each particle $X$ via scale factors $\kappa_{X}$, such that the corresponding production cross sections and partial decay widths scale with $\kappa_{X}^{2}$ with respect to the SM prediction. Here, the loopmediated couplings to gluons and photons are treated via effective coupling modifiers $\kappa_{g}, \kappa_{\gamma}$. An additional scale factor modifies the total Higgs boson width by $\kappa_{H}^{2}$. Other features of the SM are left untouched, in particular the observed Higgs boson candidate is assumed to be a single, narrow, CP-even scalar state.

Analyses are performed within several different scenarios. For example, Fig. 4 shows the result of a fit to the ATLAS data [8] assuming common scale factors $\kappa_{F}$ and $\kappa_{V}$ for all fermions and bosons, respectively. Only the relative sign of $\kappa_{V}$ and $\kappa_{F}$ is relevant here, and $\kappa_{V}>0$ is chosen to be positive.

Measuring the ratio $\lambda_{W Z}=\kappa_{W} / \kappa_{Z}$ provides a test of custodial symmetry, which within the SM implies $\lambda_{W Z}=1$. Similar ratio analyses are performed for the couplings to up and down-type fermions $\left(\lambda_{d u}\right)$ as well as to quarks

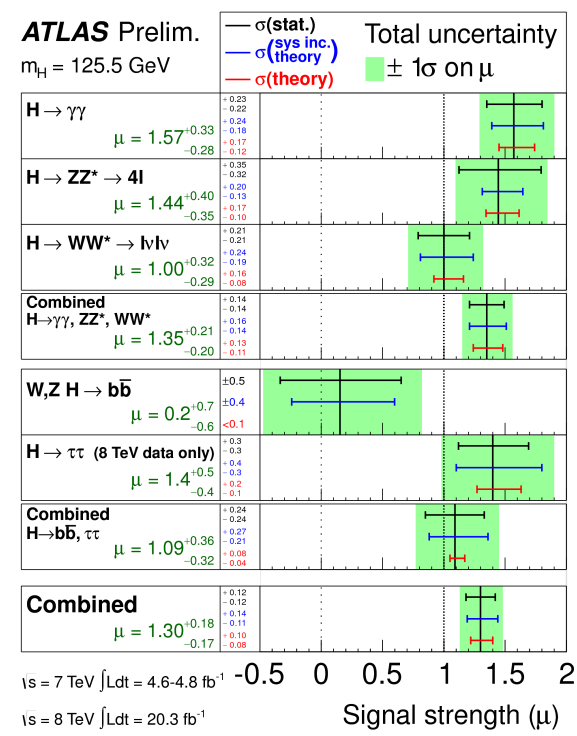

Figure 3: The measured signal strengths for a Higgs boson of mass $m_{H}=125.5$ $\mathrm{GeV}$ for the individual final states and various combinations [8]. 


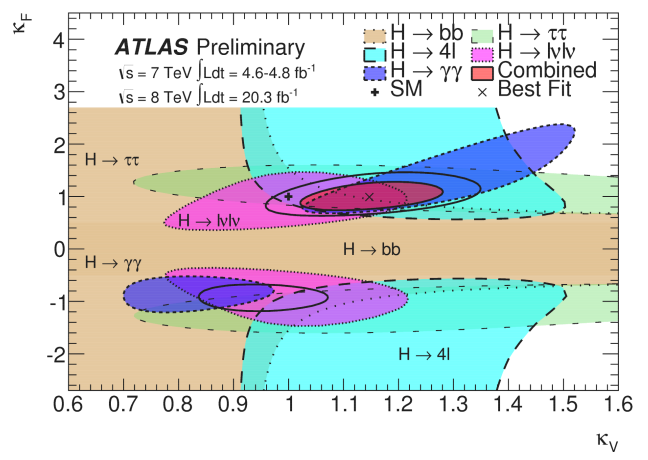

Figure 4: Results of fits for a 2-parameter benchmark model that probes different coupling strength scale factors for fermions and vector bosons, assuming only SM contributions to the total width [8].

and leptons $\left(\lambda_{l q}\right)$. Contributions from beyond-SM particles to the loops that mediate the $g g H$ and $H \gamma \gamma$ interactions are constrained by fixing all modifiers $\kappa$ to 1 except for $\kappa_{g}$ and $\kappa_{\gamma}$.

Fig. 5 provides an overview of preliminary results [8] from these Higgs boson coupling studies. While all results are consistent with the SM expectation, it should be noted that a complete, unconstrained coupling analysis will have to wait until much larger datasets will have been recorded.

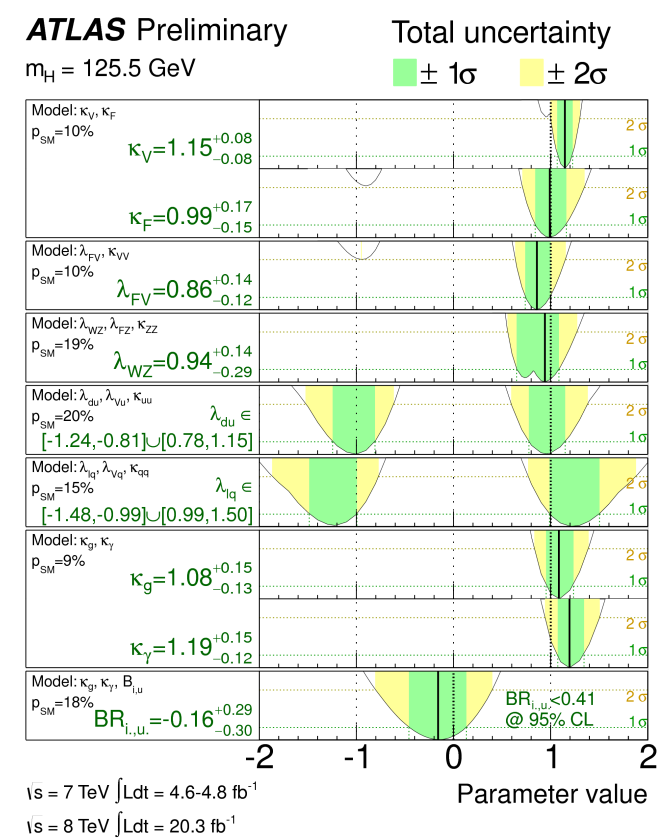

Figure 5: Summary of the coupling scale factor measurements for $m_{H}=125.5 \mathrm{GeV}$. The best-fit values are represented by the solid black vertical lines [8].

\section{Mass and Width}

At $m_{H} \approx 125 \mathrm{GeV}$, the Higgs boson is narrow and the directly observed width is given by the experimental resolution. Based on the currently available data, highresolution mass information can be obtained from two decay channels: $H \rightarrow \gamma \gamma$ and $H \rightarrow Z Z^{*} \rightarrow 4 \ell$.

From fits to the mass spectra for these decays, ATLAS measures [9] $m_{H}=125.98 \pm 0.42$ (stat.) \pm 0.28 (syst.) and $m_{H}=124.51 \pm 0.52$ (stat.) \pm 0.06 (syst.), respectively. While there is some tension between the two results, they are consistent within 2.0 standard deviations. Treating the signal strengths for the two channels as independent free parameters, the two mass measurements are combined, yielding

$$
m_{H}=125.36 \pm 0.37 \text { (stat.) } \pm 0.18 \text { (syst.) }
$$

Corresponding likelihood contours as a function of the signal yield and $m_{H}$ are shown in Fig. 6.

The mass measurements discussed above provide also constraints on the total width $\Gamma_{H}$ of the Higgs boson, which are, however, limited to a few $\mathrm{GeV}$ by the experimental resolution in reconstructing the $\gamma \gamma$ and $4 \ell$ resonances. and are thus orders of magnitude away from the $\mathrm{SM}$ prediction of about $4 \mathrm{MeV}$.

Following an alternative approach, analyses [10] of $Z Z \rightarrow 4 \ell$ and $Z Z \rightarrow 2 \ell 2 v$ events are performed in the mass range above the $2 m_{Z}$ threshold in order to obtain information on the off-shell $H \rightarrow Z Z$ signal strength $\mu_{\text {off-shell. }}$ In combination with the on-shell $H \rightarrow Z Z^{*} \rightarrow$ $4 \ell \mu_{\text {on-shell }}$ measurement, a limit on $\mu_{\text {off-shell }}$ can be interpreted as a constraint on the Higgs boson width if the

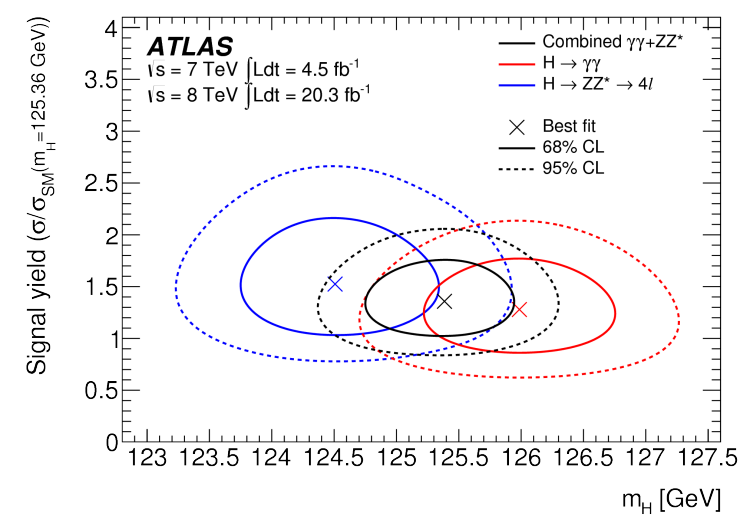

Figure 6: Likelihood contours as a function of the normalised signal yield $S=\sigma / \sigma_{S M}\left(m_{H}=125.36 \mathrm{GeV}\right)$ and $m_{H}$ for the $H \rightarrow \gamma \gamma$ and $H \rightarrow Z Z^{*} \rightarrow 4 \ell$ channels and their combination, including all systematic uncertainties. The markers indicate the maximum likelihood estimates in the corresponding channels [9]. 


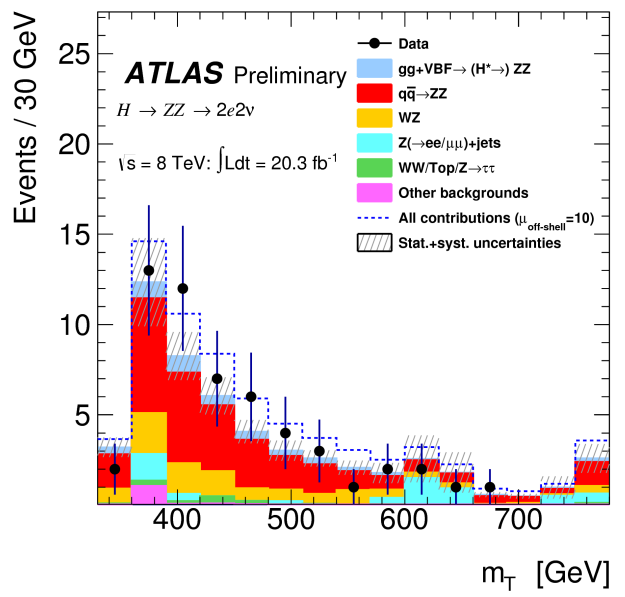

Figure 7: Observed distributions of $m_{T}$ for the $Z Z \rightarrow 2 \ell 2 v$ analysis in the signal region compared to the expected contributions from gg+VBF $\rightarrow\left(H^{*} \rightarrow\right)$ ZZ SM and with $\mu$ (off-shel) $1=10$ (dashed) in the $2 \mathrm{e} 2 v$ channel. The last bin in each distribution contains the overflow. A relative $\mathrm{gg} \rightarrow Z Z$ background $\mathrm{K}$-factor of $\mathrm{RBH}^{*}=1$ is assumed [10].

relevant off-shell and on-shell Higgs couplings are assumed to be equal.

The analyses are performed within the coupling modifier framework and the associated assumptions described above; the studied distributions depend on the channel. For example, Fig. 7 shows the observed highmass $Z Z \rightarrow 2 \ell 2 v$ transverse mass $\left(m_{T}\right)$ distribution with the expected background contributions and the predicted signal for different assumptions for the off-shell signal strength.

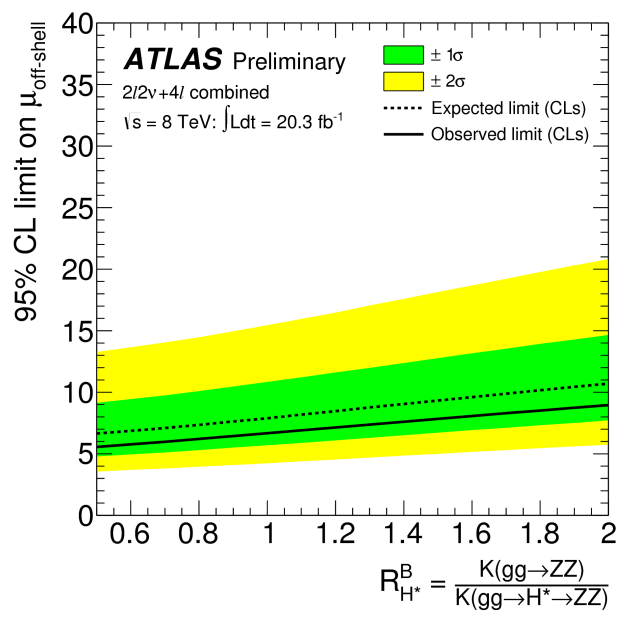

Figure 8: The observed and expected 95\% C.L. upper limits on $\mu$ (off-shell) as a function of $R_{H *}^{B}$, combining the $Z Z \rightarrow 4 \ell$ and $Z Z \rightarrow 2 \ell 2 v$ channels. The upper limits are evaluated using the CLs method including all systematic uncertainties [10].
The combination of statistical analyses in both channels yields a preliminary observed (expected) $95 \%$ C.L. limit of 5.6(6.6) $<\mu_{\text {off-shell }}<9.0(10.7)$ when varying the unknown ratio $R_{H^{*}}^{B}$ between the $g g \rightarrow Z Z$ continuum background and the $g g \rightarrow H^{*} \rightarrow Z Z$ signal between 0.5 and 2.0. This translates into 4.8(7.0) $<\Gamma_{H} / \Gamma_{H}^{S M}<$ 7.0(12.0) if identical on-shell and off-shell couplings are assumed.

\section{Differential Cross Sections}

In addition to improved inclusive analyses, the increased dataset is also used to measure fiducial and differential cross sections for events with $H \rightarrow \gamma \gamma$ [11] and $H \rightarrow Z Z^{*} \rightarrow 4 \ell$ [12] decays. For example, after correcting for detector efficiency and resolution effects, the $p p \rightarrow H \rightarrow \gamma \gamma$ cross section is measured to be [11]

$$
43.2 \pm 9.4 \text { (stat. })_{-2.9}^{+3.2} \text { (syst.) } \pm 1.2 \text { (lumi) fb }
$$

for photon pseudorapidities $|\eta|<2.37$ and transverse momenta of the leading (subleading) photon of $35 \%$ $(25 \%)$ of the di-photon invariant mass. Additional fiducial regions are defined in terms of the jet multiplicity, number of leptons and the missing transverse energy. Comparisons of the results of corresponding cross section measurements with theoretical predictions are summarised in Fig. 9.

For both di-photon and four-lepton final states differential cross sections are measured and corrected for detector effects as a function of the kinematics of the Higgs decay products and the associated jet activity. As an example, Fig. 10 shows the differential unfolded cross section in terms of the transverse momentum of the Higgs boson compared to the results of several theoretical calculations.

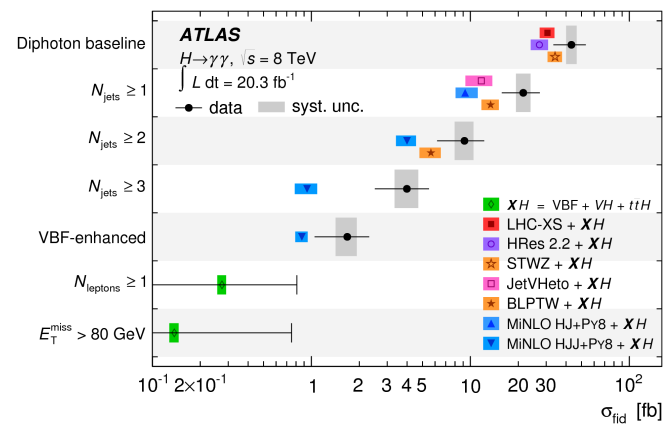

Figure 9: The measured cross sections and cross-section limits for $p p \rightarrow H \rightarrow$ $\gamma \gamma$ in the seven fiducial regions defined in [11]. The data are shown as filled (black) circles. The error bar on each measured cross section represents the total uncertainty in the measurement, with the systematic uncertainty shown as dark grey rectangles. The data are compared to state-of-the-art theoretical predictions (see [11] for details). All regions include the SM prediction arising from VBF, $V H$ and $t \bar{t} H$, which are collectively labelled as $X H$. 


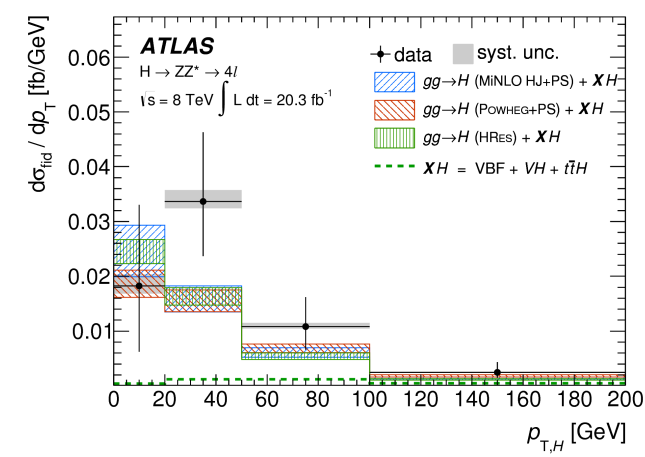

Figure 10: Differential unfolded cross section for the transverse momentum of the Higgs boson in the $H \rightarrow Z Z^{*} \rightarrow 4 \ell$ decay channel compared to different theoretical calculations [12] .

\section{Search for Top-Associated Production}

The experimental study of Higgs production in association with top quarks will eventually provide direct access to the $t H$ Yukawa coupling. It does, however, involve the analysis of complex final states and large backgrounds, in particular from inclusive top production processes. For the current preliminary ATLAS results two Higgs decay modes are considered: $H \rightarrow$ $b \bar{b}$ [13] and $H \rightarrow \gamma \gamma$ [14].

The $t \bar{t} H \rightarrow b \bar{b}$ process, which only involves Higgs boson couplings to fermions, is searched for considering fully leptonic and semi-leptonic decays of the top quark pair separately and in addition categorising the selected
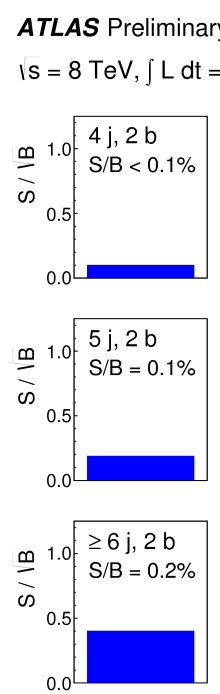

Single lepton

$\mathrm{m}_{\mathrm{H}}=125 \mathrm{GeV}$
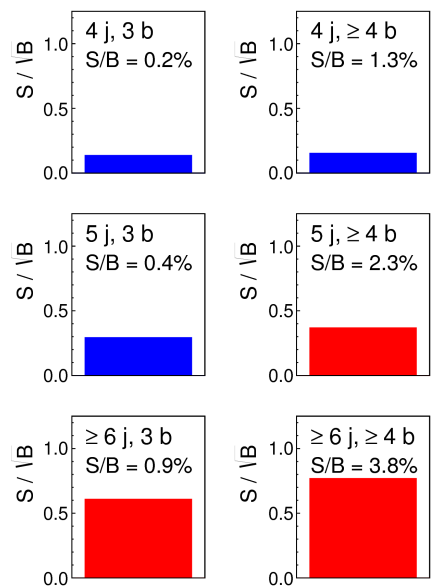

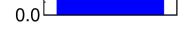

Figure 11: $\mathrm{S} / \sqrt{\mathrm{B}}$ ratio for each of the regions under consideration in the semileptonic $t \bar{t}$ channel (assuming SM cross sections and branching ratios, and $m_{H}=$ $125 \mathrm{GeV})$. Each row shows the plots for a specific jet multiplicity $(4,5, \geq 6)$, and the columns show the b-jet multiplicity $(2,3, \geq 4)$. Signal-rich regions are shown in red, while the rest are shown in blue [13].

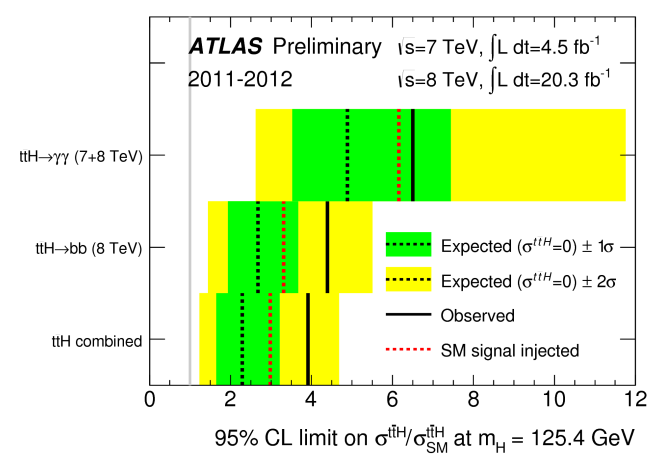

Figure 12: Observed and expected 95\% C.L. upper limits on the $t \bar{t} H$ production cross section for the individual final states and the combination [14]. The lines denoted "SM signal injected" show the expected 95\% C.L. upper limit for a datasetcorresponding to the background-plus-SM $t \bar{t} H$ production.

events in terms of the jet and $b$ jet multiplicity. Regions with large expected S/B are used as signal regions, the others provide background control samples; for the semi-leptonic $t \bar{t}$ categories this is illustrated in Fig. 11. In the signal regions, neural networks are trained to separate the signal from top backgrounds based on event shape and kinematics. A simultaneous fit to the neural network output in signal and control regions for each of the two channels and subsequent combination yields a 95\% C.L. limit of 4.1 times the SM expectation (with $2.6 \times \mathrm{SM}$ expected), which corresponds to a signal strength of $\mu=1.7 \pm 1.4$.

A combination with the results of a parallel search for $t \bar{t} H$ production via $H \rightarrow \gamma \gamma$ decays [14] improves the observed (expected) limit to 3.9(2.3) times the SM expectation, corresponding to a best-fit signal strength of $\mu_{t \bar{t} H}=1.6 \pm 0.6$ (stat. $)_{-1.0}^{+1.1}$ (syst.), see Fig. 12 .

The two-photon analysis considers also Higgs boson production in association with single top quarks, which is suppressed in the SM but can constrain also the sign of the top-Higgs Yukawa coupling. From a combination of the top-associated photon-photon analyses, observed (expected) limits on the corresponding coupling scale factor of $-1.3(-1.2)<\kappa_{t}<8.1(7.9)$ are obtained.

\section{Search for Rare Decays}

Much rarer Higgs boson decays have been searched for as well. In these cases no significant signals are expected within the SM based on the current data, and indeed none are seen. Still, these analyses are relevant both in view of possible enhancements within certain extensions of the SM and in order to prepare for future SM Higgs measurement based on larger datasets. 


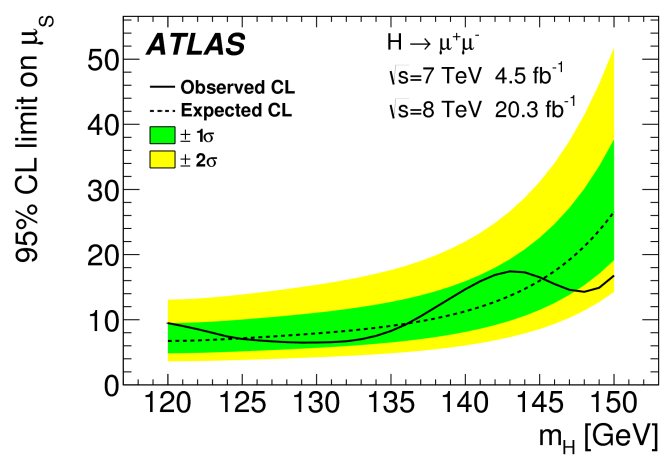

Figure 13: Observed (solid) and expected (dashed) 95\% C.L. upper limits on the $H \rightarrow \mu \mu$ signal strength as a function of $m_{H}$ over the mass range 120 $150 \mathrm{GeV}$. The dark- and light-shaded regions indicate the $\pm 1 \sigma$ and $\pm 2 \sigma$ uncertainty bands on the expected limit, respectively [15].

For example, Fig. 13 shows the upper limit on the $H \rightarrow$ $\mu \mu$ signal strength obtained from a statistical analysis of the $m_{\mu \mu}$ distributions from selected di-muon events in categories defined from the muon pseudorapidities, the transverse momentum of the di-muon system and a VBF selection. At $m_{H}=125.5 \mathrm{GeV}$ a limit of 7.0 (7.2) times the SM prediction is observed (expected) [15].

The decay $H \rightarrow Z \gamma$ is searched for [16] in events with electron or muon pairs and an isolated photon, categorised in terms of the $Z-\gamma$ pseudorapidity difference and the reconstructed Higgs boson candidate transverse momentum w.r.t. the event thrust axis. No excess over the background expectation is seen in the distribution of the di-lepton/photon invariant mass $m_{\ell \ell \gamma}$, c.f. Fig. 14, which at $m_{H}=125.5 \mathrm{GeV}$ translates into an observed (expected) upper limit of 11(9) times the SM prediction. Even first searches for di-Higgs boson production have been performed [17] based on $\gamma \gamma b \bar{b}$ final states, thus

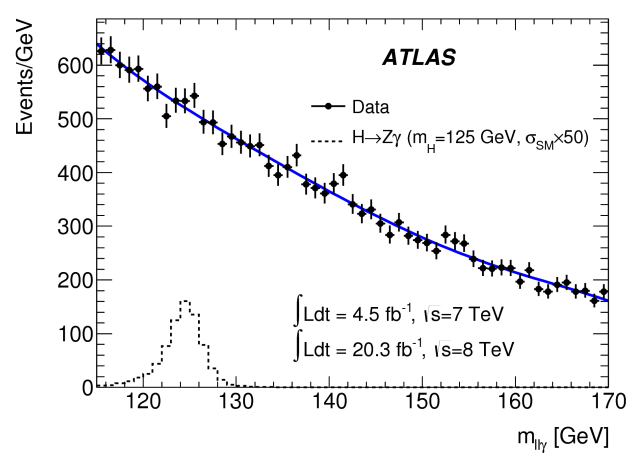

Figure 14: Search for $H \rightarrow Z \gamma$ [16]: Distribution of the reconstructed $\ell \ell \gamma$ invariant mass in data, after combining all the event categories (points with error bars). The solid blue line shows the sum of background-only fits to the data performed in each category. The dashed histogram corresponds to the signal expectation for a Higgs boson mass of $125 \mathrm{GeV}$ decaying to $Z \gamma$ at 50 times the SM-predicted rate. combining a good $m_{\gamma \gamma}$ resolutions with a large $H \rightarrow b \bar{b}$ branching fraction. Such analyses will eventually provide a measurement of the three-Higgs coupling. A 95\% C.L. upper limit on the cross section times branching ratio of non-resonant production is set at $2.2 \mathrm{pb}$, while the expected limit is $1.0 \mathrm{pb}$. The corresponding limit observed for a narrow resonance ranges between 0.8 and $3.5 \mathrm{pb}$ as a function of its mass in the range between $260 \mathrm{GeV}$ and $500 \mathrm{GeV}$, which is somewhat weaker than expected for resonances below $350 \mathrm{GeV}$.

In addition there is broad program of ATLAS analyses not covered in this overview - searching for additional Higgs bosons or using Higgs boson events as a probe for effects beyond the SM.

\section{Summary}

The release of the final ATLAS Higgs physics results from data recorded during 2011/12 at $p p$ centre-of-mass energies of 7 and $8 \mathrm{TeV}$ is progressing well, with no significant deviations from SM predictions found so far. Data taking is scheduled to resume in 2015, offering exciting prospects to continue and expand on this already rich physics program based on much larger datasets and $p p$ collision energies increased to $13-14 \mathrm{TeV}$.

\section{References}

[1] ATLAS Collaboration, JINST 3 (2008) S08003.

[2] ATLAS Collaboration, Phys. Lett. B 716 (2012) 1 .

[3] CMS Collaboration, Phys. Lett. B 716 (2012) 30.

[4] ATLAS Collaboration, Phys. Lett. B 726 (2013) 88.

[5] ATLAS Collaboration, Phys. Lett. B 726 (2013) 120.

[6] ATLAS Collaboration, ATLAS-CONF-2013-108; http://cds.cern.ch/record/1632191.

[7] ATLAS Collaboration, ATLAS-CONF-2013-079; http://cds.cern.ch/record/1563235.

[8] ATLAS Collaboration, ATLAS-CONF-2014-009; http://cds.cern.ch/record/1670012.

[9] ATLAS Collaboration, Phys. Rev. D. 90 (2014) 052004.

[10] ATLAS Collaboration, ATLAS-CONF-2014-042; http://cds.cern.ch/record/1740973.

[11] ATLAS Collaboration, arXiv:1407.4222 [hep-ex]; accepted by JHEP.

[12] ATLAS Collaboration, ATLAS-CONF-2014-044; http://cds.cern.ch/record/1741017; since then updated and submitted to Phys. Lett. B, see arXiv:1408.3226 [hep-ex].

[13] ATLAS Collaboration, ATLAS-CONF-2014-011; http://cds.cern.ch/record/1670532.

[14] ATLAS Collaboration, ATLAS-CONF-2014-043; http://cds.cern.ch/record/1740974.

[15] ATLAS Collaboration, arXiv:1406.7663 [hep-ex]; accepted by Phys. Lett. B.

[16] ATLAS Collaboration, Phys. Lett. B 732 (2014) 8

[17] ATLAS Collaboration, arXiv:1406.5053 [hep-ex]; submitted to Phys. Rev. Lett. 